\title{
Motorcycle safety device investigation: A case study on airbags
}

\author{
A CHAWLA ${ }^{1}$ and S MUKHERJEE ${ }^{1}$ \\ ${ }^{1}$ Transportation Research and Injury Prevention Program, Indian Institute of \\ Technology Delhi, New Delhi 110016 \\ e-mail: achawla@mech.iitd.ernet.in
}

\begin{abstract}
The present standard for assessing the effectiveness of safety measures for motorcycle riders defined in ISO 13232 is unique and it incorporates evaluation by computer simulation supplemented by full-scale crash tests. This report describes the process of using FE (Finite Element) simulations to investigate safety options for motorcycle riders. The experience of developing finite element models acceptable as per ISO 13232 standards has been described. The variation in dummy head accelerations on impact with inflating airbags has been studied in an environment acceptable as per ISO 13232 standards. Based on the simulations, it may be concluded that inflating airbags in motorcycles do not pose a significant injury threat.
\end{abstract}

Keywords. Motorcycle safety; finite element simulations; airbag; crash modelling.

\section{Introduction}

Van Driessche (1994) describes development of ISO/CD 13232, which specifies test and analysis methods for research evaluation of rider crash protective devices fitted to motorcycles. It is not a safety standard or a law but provides a methodology by which assertions regarding safety efficacy of proposed devices needs to be evaluated to be accepted by the scientific community. We summarize the ISO committee process involving experts from 10 nations, at the request of United Nations ECE/TRANS Working Party 29. The Standard was subsequently approved worldwide as ISO 13232 (1996). The ISO Standard provides a set of common requirements and assumptions for minimum levels of modelling detail, parameter measurement, output variables, post-processing (in terms of three-dimensional animations and injury indices), quantitative (rather than qualitative) calibrations, correlations and comparisons against recorded and simulated test data.

Since simulations are part of the standard, the calibration procedures in the Standard are intended to enable simulations to use data interpolated between conditions that have been tested in full-scale or in laboratory, but not extrapolated beyond the tests. The purpose of the simulation tool, and the ISO Standard itself, is to assess the relative injury benefits and risks of protective devices across a large (200) representative cluster of conditions reported in real accidents, a task which is logistically infeasible by means of full-scale-tests (FST). The 
standard hence mandated only seven full-scale tests to act as validation points. Since this was a motorcycle (MC) standard, the other vehicle (OV) was standardized to be a 1989 Toyota Corolla and a specialized Motorcycle Anthropomorphic Test Dummy (MATD) was to be used. The safety evaluation is then based on simulation of the 200 impact types. The Standard is not intended to be either a workbook or user manual for 'how to' implement a motorcycle crash simulation, but rather a standard which ensures that minimum levels of detail, performance and calibration are used, so that the results of the overall analysis may be relied upon.

Since this is a simulation based standard, it is of interest to trace the historical development of MC simulation. A two-dimensional multi-rigid-body Lagrange formulation of a five mass rider and a single mass motorcycle was used to model rider/motorcycle/barrier impacts by Knight \& Petersen (1971) and summarized in Bothwell \& Petersen (1971). HappianSmith et al (1987), Happian-Smith \& Chinn (1990), Chinn \& Hope (1987), Chinn et al (1989) describe 2D lumped mass models developed to study MC rider safety. Sporner (1982) described a 10 degree of freedom, 2D MB model. Subsequently, Bothwell et al (1973) and Knight \& Petersen $(1973,1976)$ report 3D multi-body (MB) models of the motorcycle. Nieboer et al (1993), Chinn et al (1996) and Deguchi (2003) reported airbag simulations using a multi-rigid-body MADYMO simulation with finite element (FE) airbag model. Yettram et al (1994) and Wang and Sakurai, 1999 are two other approaches to motorcycle model development. Chawla et al (2001) describe the first complete FE model for the MC and the car. Consequently, the standard is based on the state-of-the-art of 1996, MB models. Present day FE simulations can be of much higher fidelity and built more easily from CAD models and material properties by manufacturers.

Development of car-motorcycle crash models in the ISO 13232 configurations were conducted by Zellner et al (1994) using a 3-dimensional multi-rigid-body model based on the ATB code which was applied to 163 impact configurations based on groupings of accidents in LA and Hannover, the same set from which the ISO 13232 standards evolved. Rogers (1994) describes simulations of rider injuries with a baseline and a modified sports motorcycle. Zellner et al (1994) describe extensions of the Zellner et al (1994) simulation model, including a control volume airbag, an airbag mechanical sensor model, an igniter time delay, separate helmet mass, deformable chest and abdomen models, and a refined injury cost model. Kebschull et al (1998) describe simulation comprising a 7 mass motorcycle, a 30 mass MATD dummy and a 7 mass car. The model was validated against experimental data and was subsequently applied to the 200 LA/Hannover impact configurations. Iijima et al (1998) describe a hybrid $\mathrm{FE} /$ multi-body simulation involving the LS-DYNA3D ${ }^{\mathrm{TM}}$ and ATB codes. This comprised a 7 mass motorcycle, a 614 element FE airbag, a 30 mass dummy and a 7 mass car. Canaple et al (2002) describe a multi-rigid body MADYMO ${ }^{\mathrm{TM}}$ model of a motorcycle, dummy and car, used to generate head acceleration time histories for input to a finite element model of a human head and brain. The car was a rather unique multi-body model involving 25 or more rigid-body masses, modelled by a combination of physically cutting up and measuring various structural elements and by calculating force-deflection characteristics based on sub-structure FE modelling. These are case studies using ISO 13232 configurations but do not report the conformity to individual sub-sections. Rogers et al (2005) describe the methodology that can be used to develop motorcycle simulations as per ISO13232.

Chawla et al (2001) describe one of the first complete FE models for the MC and the car, developed using the PAMCRASH ${ }^{\mathrm{TM}}$ software. Mukherjee et al (2001) and Nakatani et al (2001) describe this model in more detail. Chawla et al (2003) compared the kinematics of the FE simulation with recording of ISO 13232 car-front impact tests to evaluate the qualitative fidelity. Deguchi (2003) describes a hybrid FE/multi-body MADYMO ${ }^{\mathrm{TM}}$ model comprising 
a 21 mass motorcycle, 2200 membrane element airbag, a hybrid III dummy for crash into a rigid barrier. Namiki et al (2003) describes a hybrid FE/multi-rigid body model using the LS-DYNA $^{\mathrm{TM}}$ code, comprising a $35 \mathrm{~K}$ element motorcycle, a $5 \mathrm{~K}$ element airbag, a $36 \mathrm{~K}$ element dummy and a $169 \mathrm{~K}$ element car to evaluate the efficacy of the airbag.

This report discusses the process of creating an FE based simulation that satisfies the requirement of the ISO 13232 standards and its use in evaluating airbags. Separate parts of the motorcycle were modelled in FE and tested under static loading and impact tests as per ISO standards for validation. The full motorcycle model when assembled from the validated components resulted in the first full scale FE model of a MC. This was evaluated by comparing the kinematics and wall forces measured from a full-scale barrier test. A model of the Toyota Corolla built using a similar process was then used for simulations for carmotorcycle crashes. The simulations achieved a high level of fidelity and allowed study of phenomenon not easily observed in experiments suggesting that motorcycle crash results may not be as graded as car crashes have been. Some phenomena from these simulations have been highlighted as an indicator to the level of completeness or fidelity that the FE simulations can achieve.

Having established a motorcycle model compatible to ISO 13232 standards, workon suitability of the airbags in small $100 \mathrm{cc}$ MCs commonly used in India has been initiated. The role of airbags in mitigating the effect of impacts is well established. The special problem in MC airbags is that the rider position is not as standardized as the seat belt strapped car rider. Therefore, the inflation stage of the airbag is of significant concern. The ALE (Arbitrary Lagrange-Euler) approach was seen to correctly predict experimentally observed airbag deployment sequences. The class of injury sustained by the rider due to contact with the airbag during its deployment phase is shown to have a low injury probability and variation due to the initial distance and the airbag size variation for practical airbags is not significant. Hence airbags can be designed for motorcycle safety without great concern for inflicting injury to out-of-position riders.

\section{Car-motorcycle crash simulation - An overall methodology}

Chawla et al (2001) describe details of the methodology used to develop the MC simulation models. Simulation models have been developed for the Toyota Corolla, 1989 model car which was the ISO standard and the Kawasaki GXZ, 1988 model motorcycle for which full-scale test data was available. The car and the motor cycle manufacturers did not provide geometry or material data because of legal requirements. A reverse engineering approach was therefore adopted.

A finite element mesh was built for sub-sections of the car and motorcycle using the geometry obtained from a coordinate measurement machine. A relatively denser mesh was created for components expected to undergo large deformations during impact than components expected to have little or no deformations. Impact tests as per ISO13232 standards were conducted on components that played important roles in determining the kinematics and dynamics of the rider. The displacement, velocity, acceleration and force histories were recorded as function of time for quasi-static as well as impact loading. Figure 1 shows the set-up used in one such test for developing the validated model of the fuel tank Chawla et al (2001). The static simulations were done in Ideas and dynamic simulations were done in PAMCRASH ${ }^{\mathrm{TM}}$ to validate the component.

For simulation in PAMCRASH ${ }^{\mathrm{TM}}$, the file containing the meshed description of the geometry was built in IDEAS ${ }^{\mathrm{TM}}$ and transferred to PAMCRASH ${ }^{\mathrm{TM}}$. Boundary conditions and 


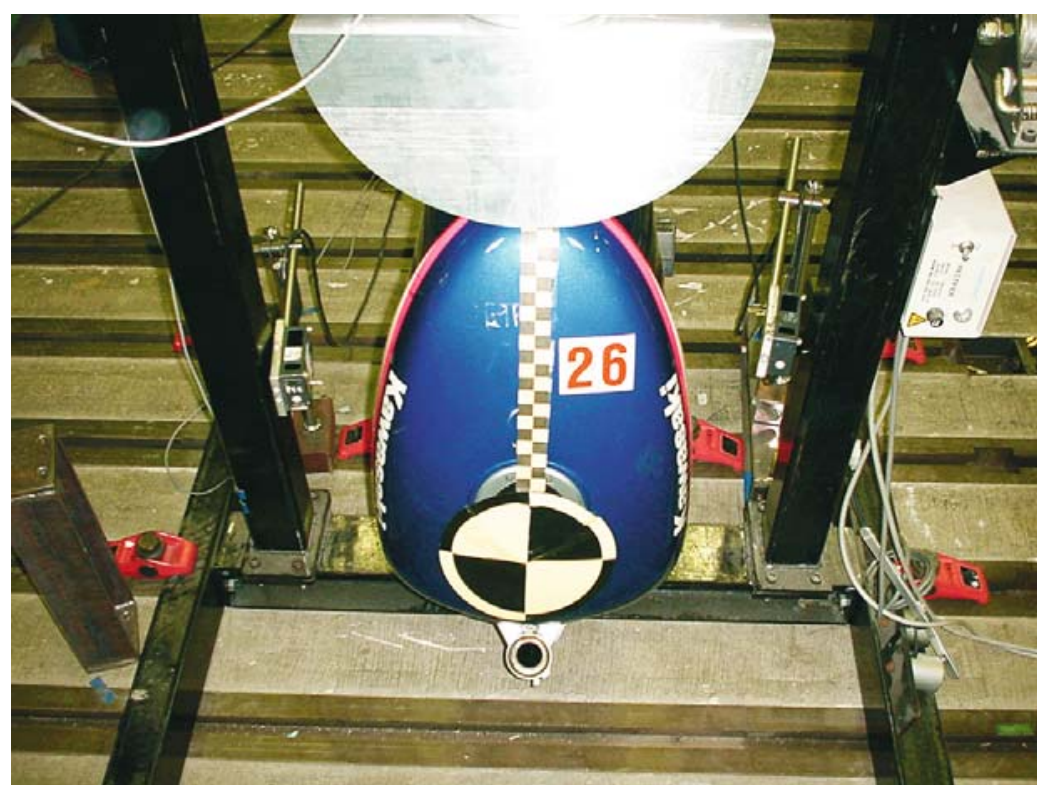

Figure 1. Set-up for performing a drop test for motorcycle fuel tank.

material properties were then defined. Figure 2 compares the experimental and simulated time history for the dynamic drop test done on the fuel tank Chawla et al (2001).

The geometry could be determined to reasonable accuracy from coordinate measurements. To estimate the material properties, extension tests on universal testing machine and vibration tests to estimate the Young's modulus of the material were conducted. In some cases where material testing was not possible, an inverse mapping method was used to tune the component model to match with experimental results. The component tests thus helped in validating the models as well as in estimating material properties. Figure 3 shows a typical simulation where the windscreen model was validated for the impact of the windshield with helmet and impactor. Mukherjee et al (2001) describe these simulations in more detail.

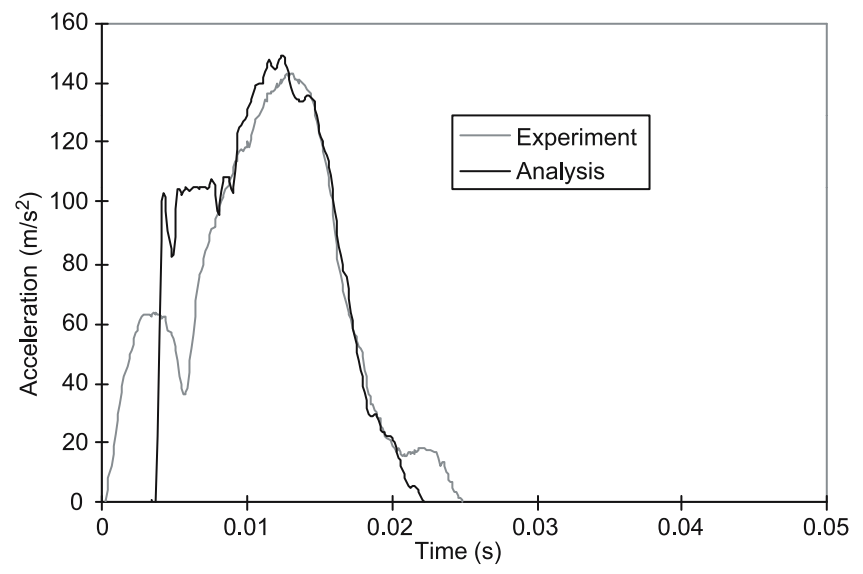

Figure 2. Acceleration-time curves of experimental and computer simulation results in drop test to a tank. 


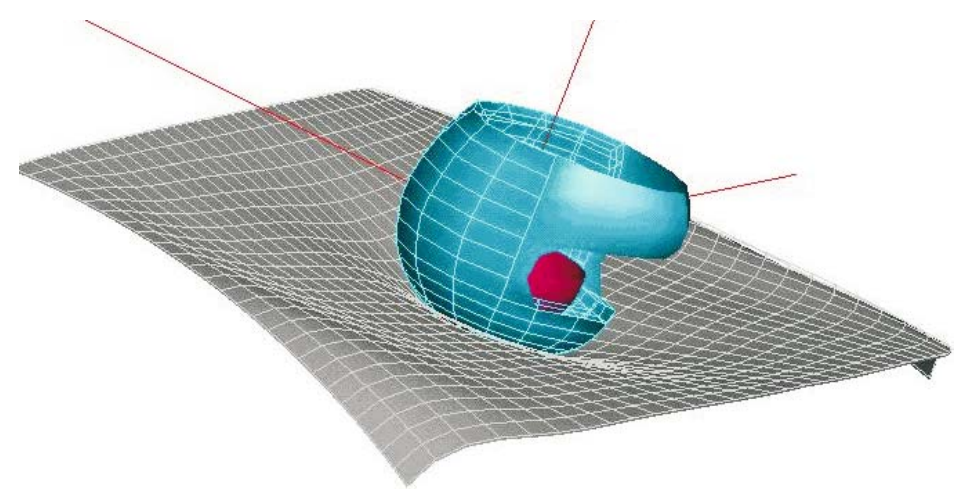

Figure 3. Simulation of dynamic impact on windscreen with helmet and impactor.

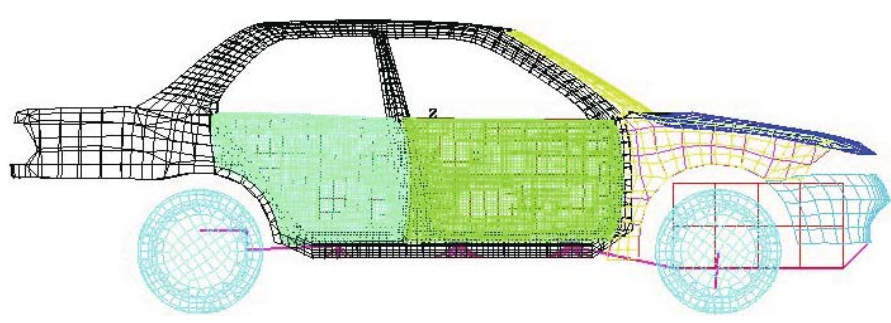

Figure 4. Finite element model of the car used to simulate the impact with the motor cycle.

The validated components were assembled to complete the finite element model of the car and the motorcycle (figures 4 and 5). These models were then used to simulate the car - MC side impact crashes Chawla et al (2001). Beam elements were used to model the frame of the motorcycle using digitized data and measured cross-sections. The tire of the motorcycle was modelled according to methodology developed by Takatori (1999) with shell elements for

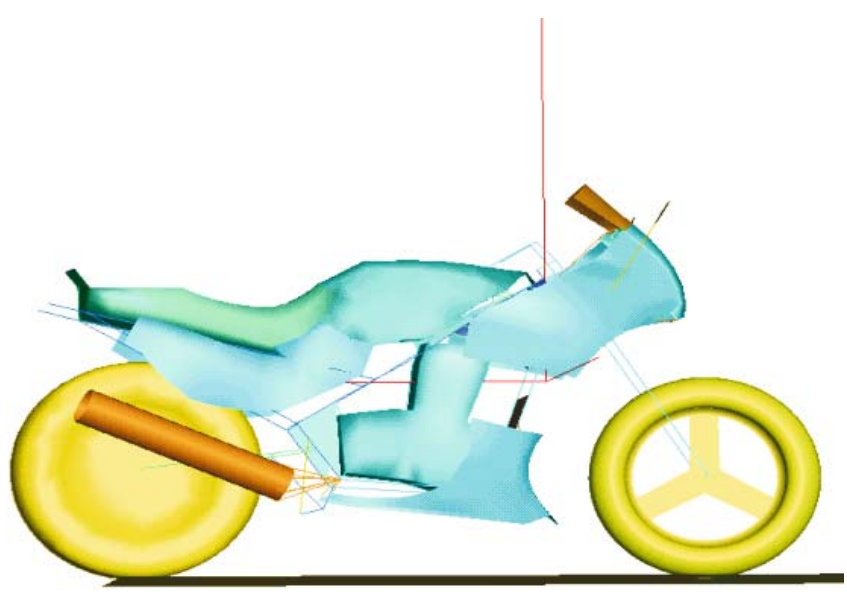

Figure 5. Motorcycle model. 
tread and side wall and brick elements for the rim of motorcycle. Static internal pressure of $200 \mathrm{Kpa}$ was defined in the tire. Other parts of the motor cycle like the fuel tank, engine, seat, etc. have been modelled using shell/solid elements. The front suspension, rear suspension and the hinges are modelled using joints which allow definition of stiffness and damping. Nodal masses have been selectively added to adjust the overall mass, center of gravity and moment of inertia of different segments of the motorcycle. Figure 5 shows the model of the MC.

The car-MC impacts also require the stiffness and attenuation properties of the suspension of both the vehicles. These data were not available and were estimated from experiments. The front and the rear suspensions were isolated and the load-deflection characteristic was measured to estimate stiffness of the suspension. The suspension damping was estimated by applying the exponential decay law to the deflection-time plot obtained when the vehicle was initially loaded and then released suddenly.

\section{Motor cycle model validation}

As a precursor to the simulation of car-motorcycle crash simulation, the motorcycle model was independently validated through the simulation of a motorcycle-wall crash at an initial velocity of $47.6 \mathrm{Km} / \mathrm{h}$ (Mukherjee et al 2000). Recording of the wall forces during the crash, visual comparison of the kinematics and the acceleration at center of gravity of motorcycle node, node on the bottom of the seat were used to validate the model.

For the frontal motorcycle wall crash the wall was modelled as rigid and fixed. The road was defined as rigid and motorcycle was allowed to run (in simulation) on the rigid road to let the tires spin-up and to stabilize the deflections in the shock absorbers. Figure 6 shows a comparison of the kinematics of the experimental crash with that of the simulation. The kinematics in the two cases as seen on the timing of key features like wheel lift-off and rotation compare very well. In addition, the peak forces measured at the wall shown in figure 7 compared very well. However, the structural vibrations were not reproduced.

\section{MC-car side impact simulations}

The validated MC and car models have been used for simulating the car-MC crashes in 7 different configurations. The configurations specified in ISO13232 were developed on the basis of the MC model validation (figure 8). Mukherjee et al (2001) describe the simulations developed for the situation when the car is impacted on the side by the MC.

The grip of the palm on the handle of the motorcycle affects the tests and the way they are modelled affects the simulations in a significant way (Mukherjee et al 2001). Initially, contact interaction was not defined between the palm of the dummy and the handle of the motorcycle to model the fact that the grip is lost on impact. The hand of the MATD dummy (specially modified for motorcycle simulations) used for the crash test has an encircling grip on the handle. It was determined through measurements that it requires a force of about $250 \mathrm{~N}$ to open the grasp. Consequently, the dummy continues to hold on to the handle of the MC well after the MC impacts the car. This effect was verified by studying crash test film. Simulations with the stronger grip of the dummy hand modelled with handle-hand contact extending to $100 \mathrm{~ms}$ lead to the dummy head gaining additional $30 \mathrm{~mm}$ in height.

The kinematics of the motorcycle, rider and helmet are reproduced well in the simulations. The point of impact of the head in the experiment is near the top sill. The contact location 


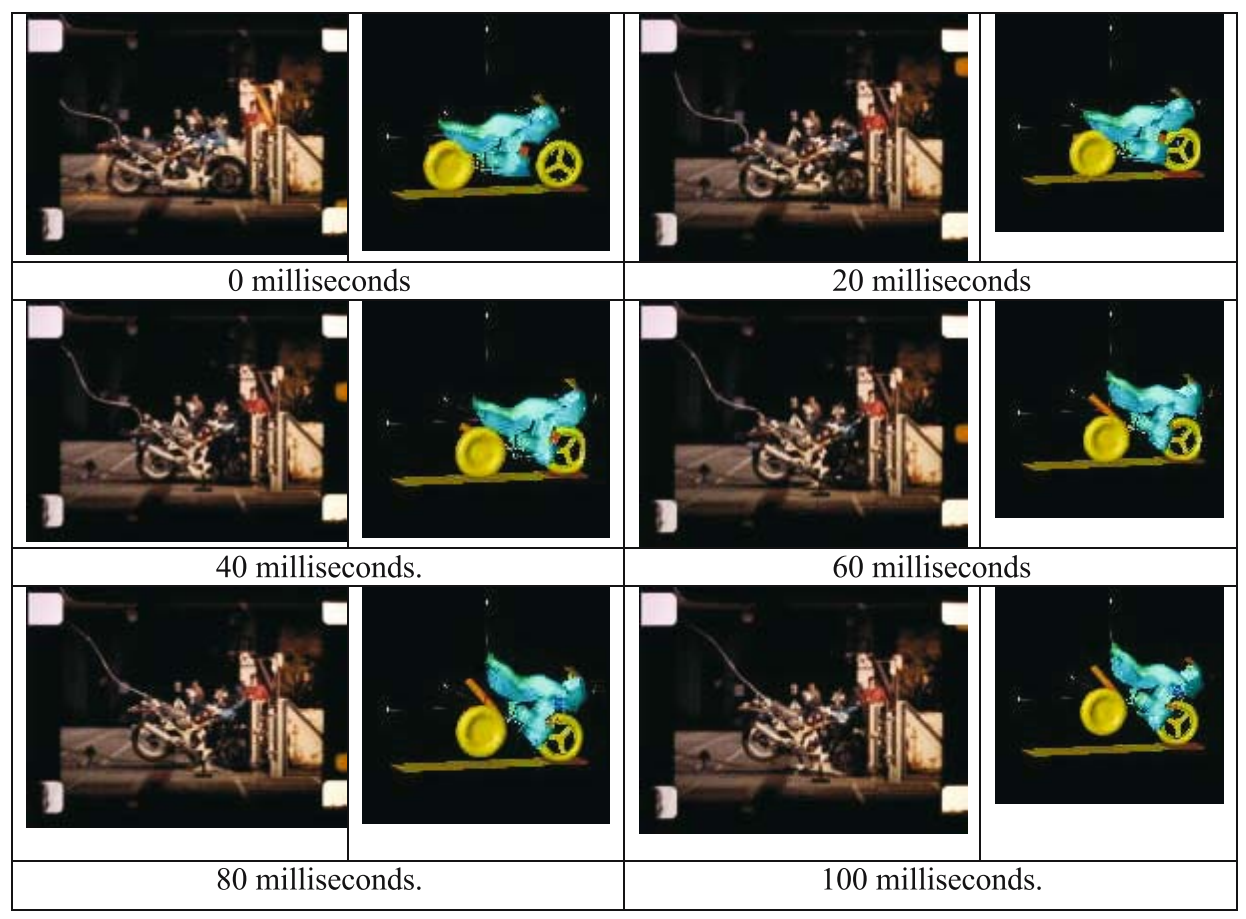

Figure 6. Comparison of motorcycle crash results in the experiment and in simulations in impact with a wall.

can be very critical and the end result can be the helmet sliding on the roof or getting lodged on the sill due to a variation of as little as $30-50 \mathrm{~mm}$ in the impact height (figure 9). The simulations hence indicate that small changes in the configuration could lead to significant variation in the head injury parameters (Mukherjee et al 2001).

The other side-impact cases reported in Mukherjee et al (2001) also show a close qualitative match with the experimental observations. The simulations showcase the effects of some

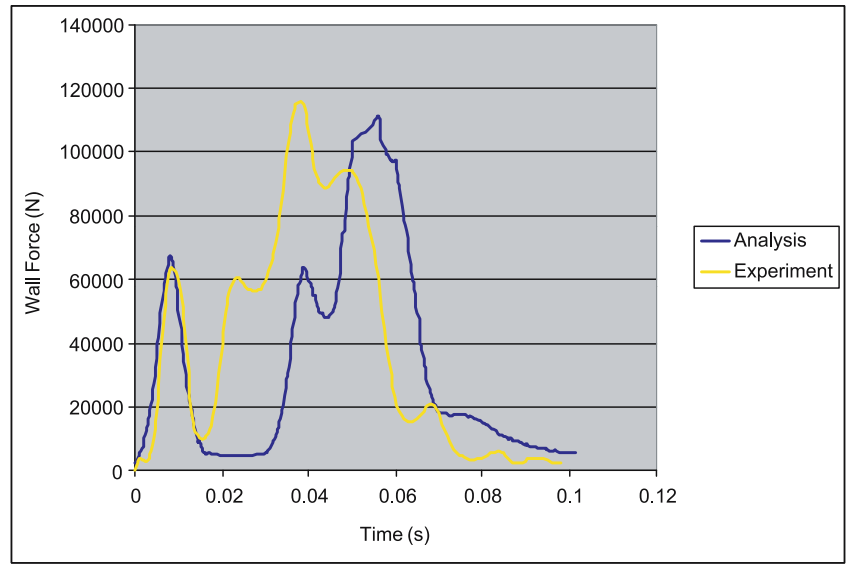

Figure 7. Force-time curves measured for motorcycle impact with a wall. 

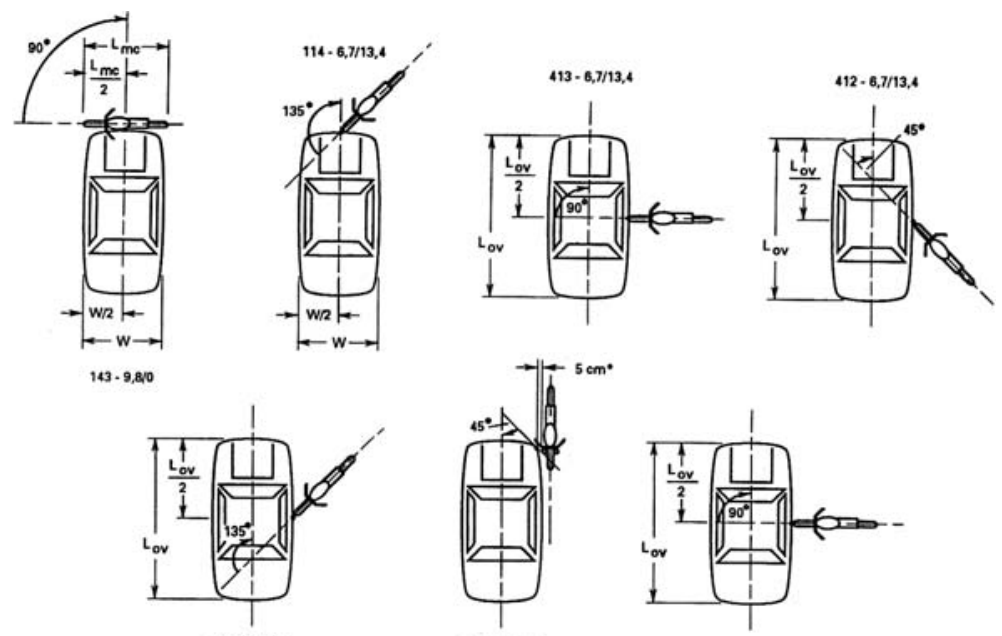

$414-6,7 / 13,4$

$225-0013,4$

$$
413 \cdot 0 / 13,4
$$

Figure 8. Motorcycle impact configurations provided in ISO13232.

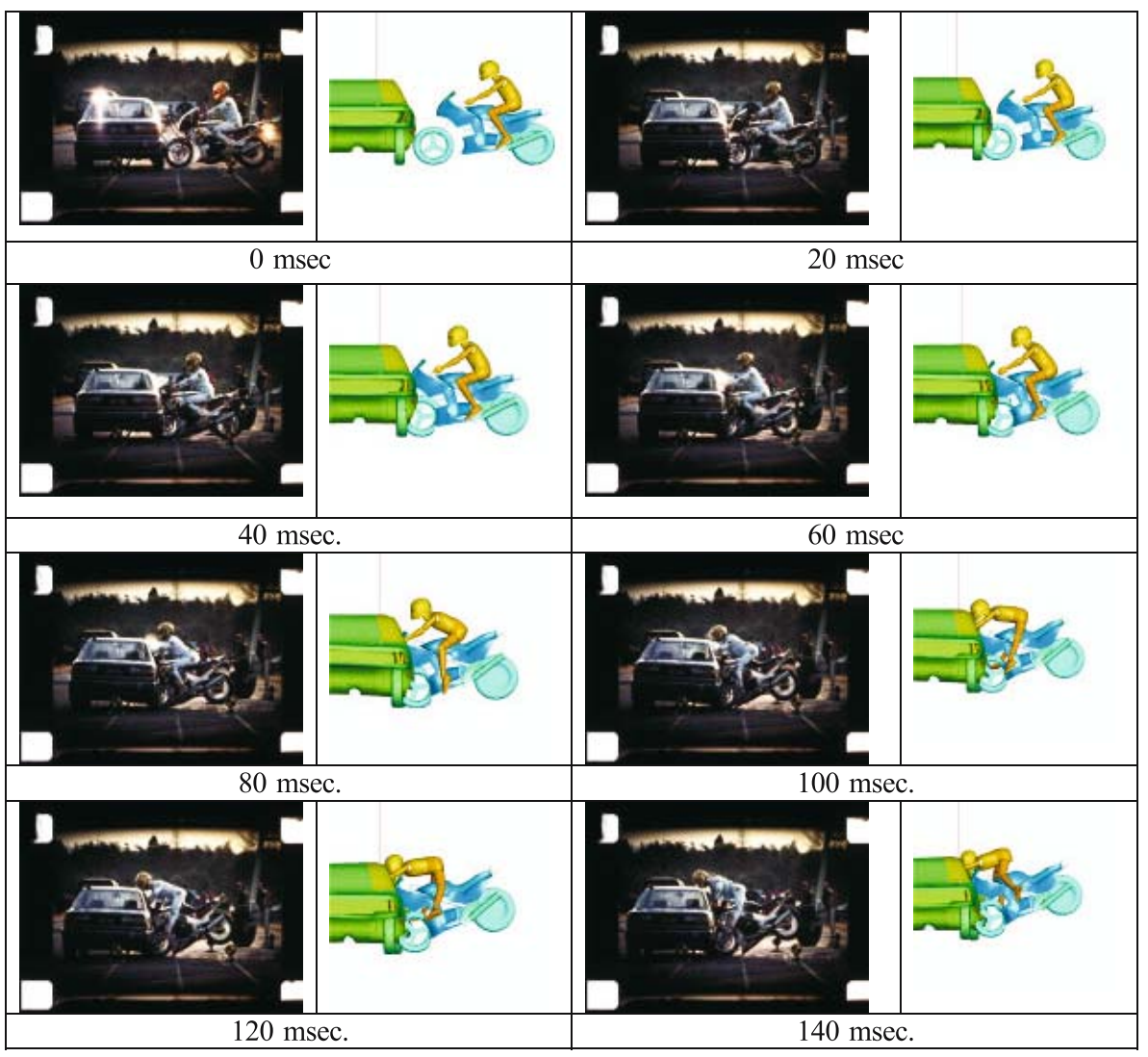

Figure 9. Comparison of the simulation kinematics with the motorcycle crash test at $20 \mathrm{msec}$ intervals. 
more differences between the H-III and the MATD dummy which play an important role in the outcome of car-MC impacts. Mukherjee et al (2001) also detail that some configurations listed in [ISO13232] are very sensitive to the impact location.

\section{FE simulations of motorcycle - car-frontal crashes}

Chawla et al $(2004,2004 \mathrm{c})$ detail simulations developed to model impacts of the motorcycle with the front of the car. Figure 10 shows snapshots of the simulation alongside that of the experiment. In both the simulation as well as in the full-scale test (FST) the dummy starts bending from the initial vertical position at around $80-90 \mathrm{~ms}$ (figure 10). The dummy head moves toward the edge of the bonnet/windshield, but the head impact is avoided as the $\mathrm{MC}$ and the dummy start moving away from the other vehicle (OV) due to the impact of the body. The inclination of the dummy and the height gained by the right leg is greater in the simulation. This disparity could be attributed to the Hybrid-III joint structure being different from that of the MATD dummy. Of the variations present in the MATD upper body structure, one of the most significant features is that the MATD neck allows a twist that is restricted in the Hybrid-III. In general the MATD joints are tightened more than the Hybrid III joints during assembly to maintain stability in the run up to the impact (ISO13232). Similar variation between simulation and experiment in the head movement in spite of consistent torso movement is seen in other cases as well (Chawla et al 2003).

Figure 10 compares the kinematics in the simulations with those in the experiment as discussed in Chawla et al (2004). The leg protector (LP) is the first MC component to contact the bonnet. In the absence of the LP, the fuel tank and the front portion of the MC would establish the initial contact. During impact, the bonnet bending near the midline is of significance. This design of the bonnet is to stop the pedestrian from impacting the hard areas near the windscreen in case of an impact. During modelling, care was taken to capture the curvature in the bonnet (and the resulting bending) correctly. We argue that the bending characteristics of the bonnet should be validated for realistic results as part of the standards (Chawla et al 2004).

Figure 10 shows that the MC-OV impact starts with the left LP coming in contact with the bumper, and this instant of time is marked as $t=0$ as shown in the experiment. At $20 \mathrm{msec}$, the left LP strikes the bonnet hood. From $50 \mathrm{msec}$, the bonnet hood starts to bend from the middle and forms a ' $\mathrm{V}$ ' shape. The bending in the simulation is more prominent and progresses somewhat faster than in the FST. From $60 \mathrm{~ms}$, the left arm loses contact with the handle and the dummy falls over the bonnet.

It was found that detailed modelling of the bumper, the radiator and the bonnet for the OV, and the silencer, fuel tank and the shock absorber of the motorcycle are critical. Nodal sticking is a known phenomenon in FE simulations, wherein the node is assumed to have approached the interacting surface from the 'wrong' side. In MC-OV simulations this problem is quite acute because of the complexity introduced due to large deformations and the significant number of sharp edged surfaces being present. This is not as significant an issue in car simulations as most exposed surfaces have now been re-designed with smooth contours for safety.

At this stage, the extensive quantitative comparison of the accelerations and the injury indices mandated by ISO 13232 has not been attempted. From a research perspective, attempting a quantitative comparison of accelerations and injury parameters is relevant only after a qualitative match can be established in the kinematics. Simulations indicate that some of the impact configurations might be hypersensitive to parameter variation. On the basis of 

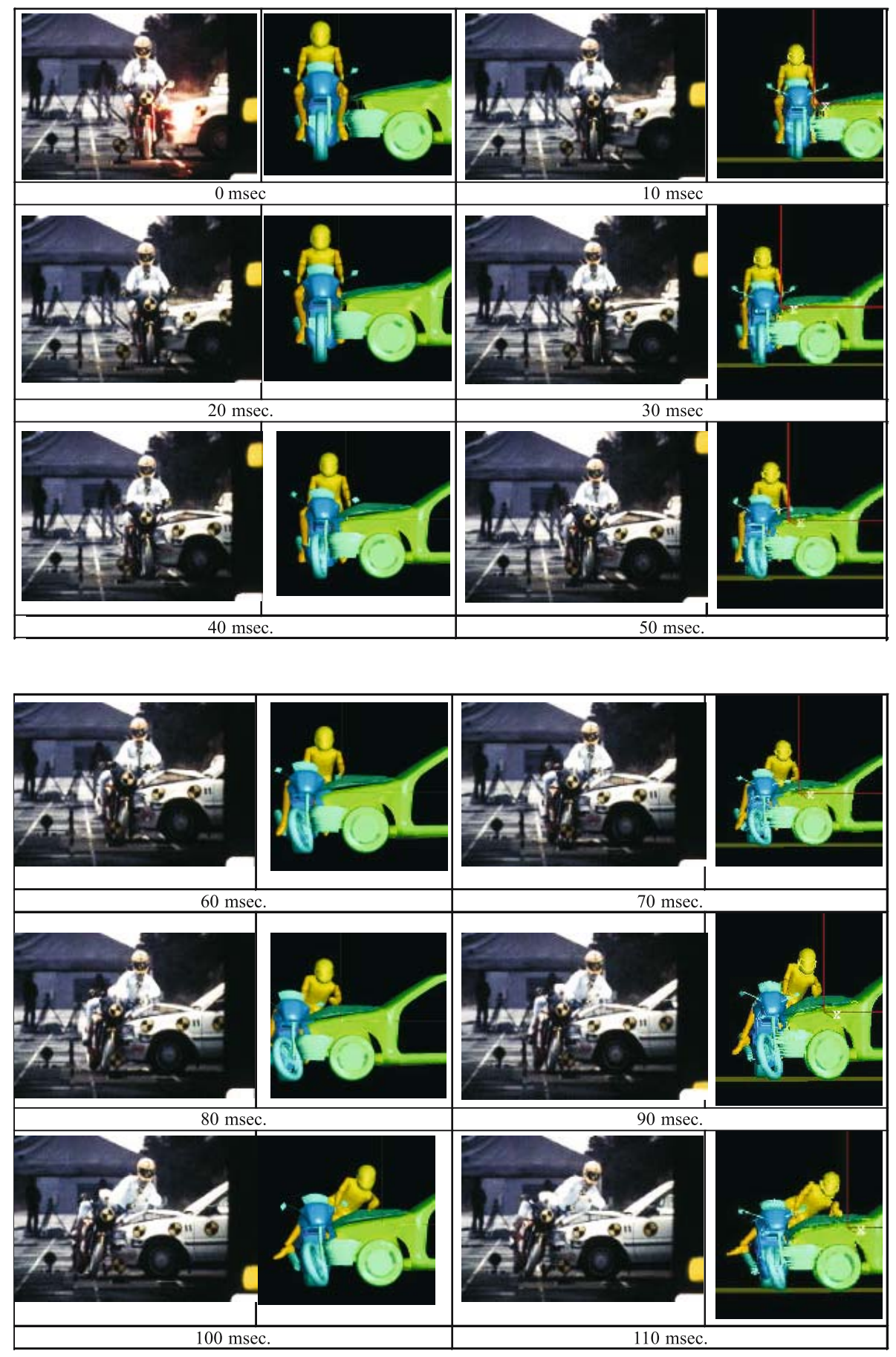

Figure 10. Comparison of the kinematics of the experiment and simulation. 


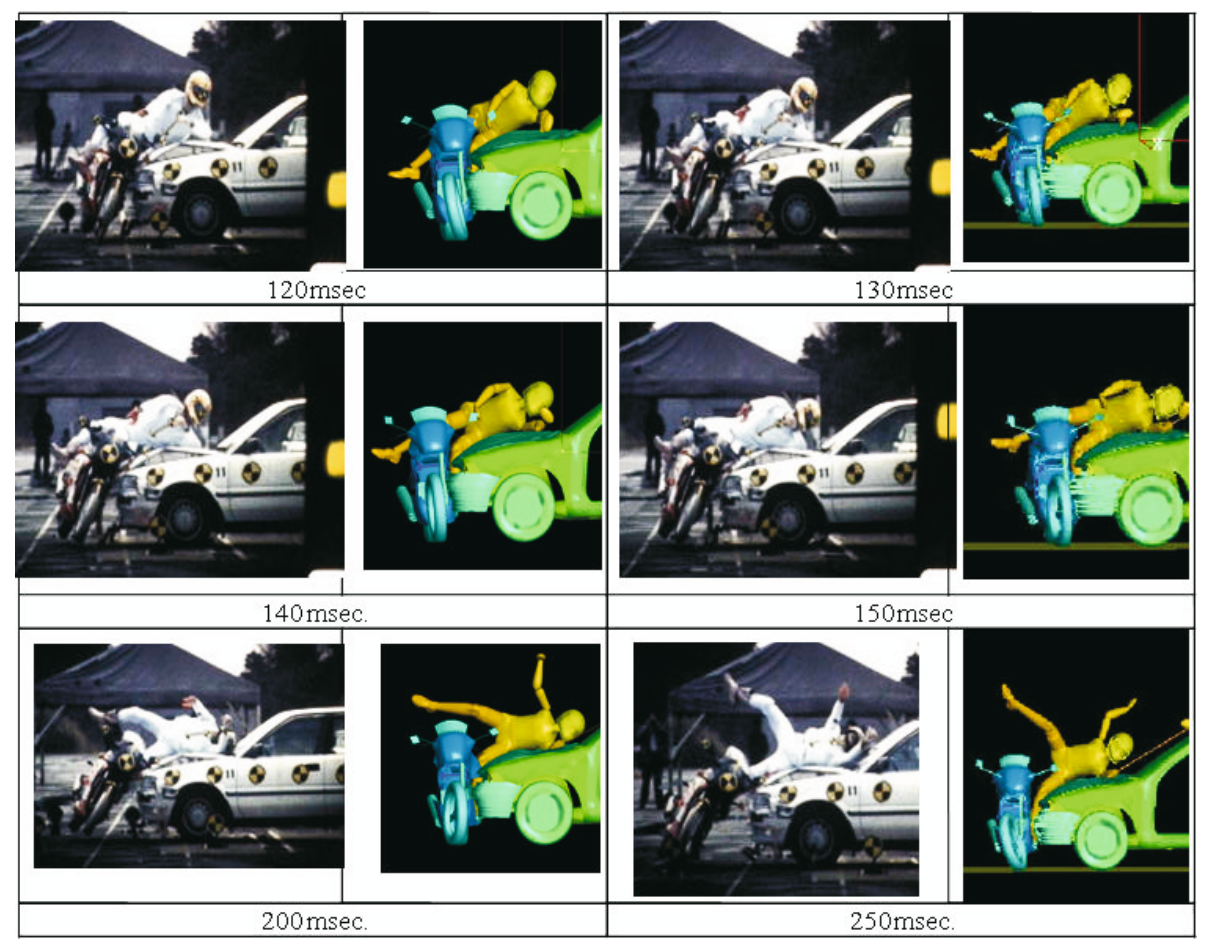

Figure 10. (Continued)

these simulations it is felt that the repeatability/reproducibility of these impact configurations should be investigated (Chawla et al 2004).

\section{Studies on motor cycle airbags}

One of the safety systems in consideration for motorcycles, of late, has been the deployment of airbags Ijima et al (1998) motivated by the success of airbag technology in automobiles. The issues which make MC airbags different from automobile airbags are the presence of a helmeted rider, the rider being unbelted and hence not confined in space, varying size and posture of riders, exposure to outside environment, etc. (Rogers \& Zellner 2001). Recently, studies have been reported where airbags were tried for large touring motorcycles with engine volume capacity of more than $600 \mathrm{cc}$ (Watson \& Donne 1989); (Iijima et al 1998). The Transport and Road Research Laboratory (TRRL), United Kingdom has conducted some experiments on lightweight motorcycle called Experimental Safety Motorcycle4 (ESM-4) with 1301 size airbag (Watson \& Donne 1989). They found that airbags can also be accommodated in small $125 \mathrm{cc}$ motorcycle. Honda has announced the commercial availability of airbag with their $1800 \mathrm{cc}$ motorcycle [www.worldnews.honda.com]. Thus, it has taken more than three decades of research on motorcycle airbags to establish the feasibility of airbags at least for large touring motorcycles Kuroe et al (2005). However, issues in airbags for small $(\sim 100 \mathrm{cc})$ motorcycles, mostly of concern only to developing countries in SE Asia, where these MCs are popularly used as a means of transport have not been addressed. 
One of the limitations perceived in deploying airbags is that two wheeler riders are less likely to be in a fixed location with respect to the airbag at the point of impact, called the 'out of position' (OOP) effect. We have developed computer simulations to study the OOP effect which has been a significant factor in cars.

Though computationally more expensive than the traditional Control Volume (CV) approach, an Arbitrary Lagrange-Euler (ALE) airbag model (Cirak \& Radovitzky 2003); (Haufe et al 2004); (Marklund \& Nilsson 2002) is able to simulate the initial stages of deployment better when compared to experimentally measured airbag shapes during deployment. The control volume approach typically underestimated the airbag pressure during the initial stages of deployment and consequently underestimated the injury that might be caused when the head is too close to the airbag. The novelty of the current work lies in the study of airbags when the rider's head is very close to the airbag, using ALE approach with rider positions compatible to ISO 13232.

In order to study the effect of varying head distances, a series of simulations were run by varying the initial distance between the dummy and the airbag from $50 \mathrm{~mm}$ to $300 \mathrm{~mm}$. Instead of freely positioning the head, the bend of the rider was made compatible to that of a seated Hybrid III dummy. This also determined the orientation of the head. An experimentally validated airbag model was the starting point. The airbag was scaled to form volumes from 1201 to 1801 and the inflator characteristics adjusted such that the peak pressure in the free expansion of airbag remained the same, for all the volumes. This gave a peak mass-flow rate for the 1201 airbag as $3.3 \mathrm{~kg} / \mathrm{s}$, and for the 1801 airbag it was $3.9 \mathrm{~kg} / \mathrm{s}$. The setup of the simulation is shown in figure 11. The first significant step in this process is the development of the FE mesh of the folded airbag. The techniques used have been described in Chawla et al (2004a), Chawla et al (2004b), and Chawla et al (2005) and are not included here for brevity.

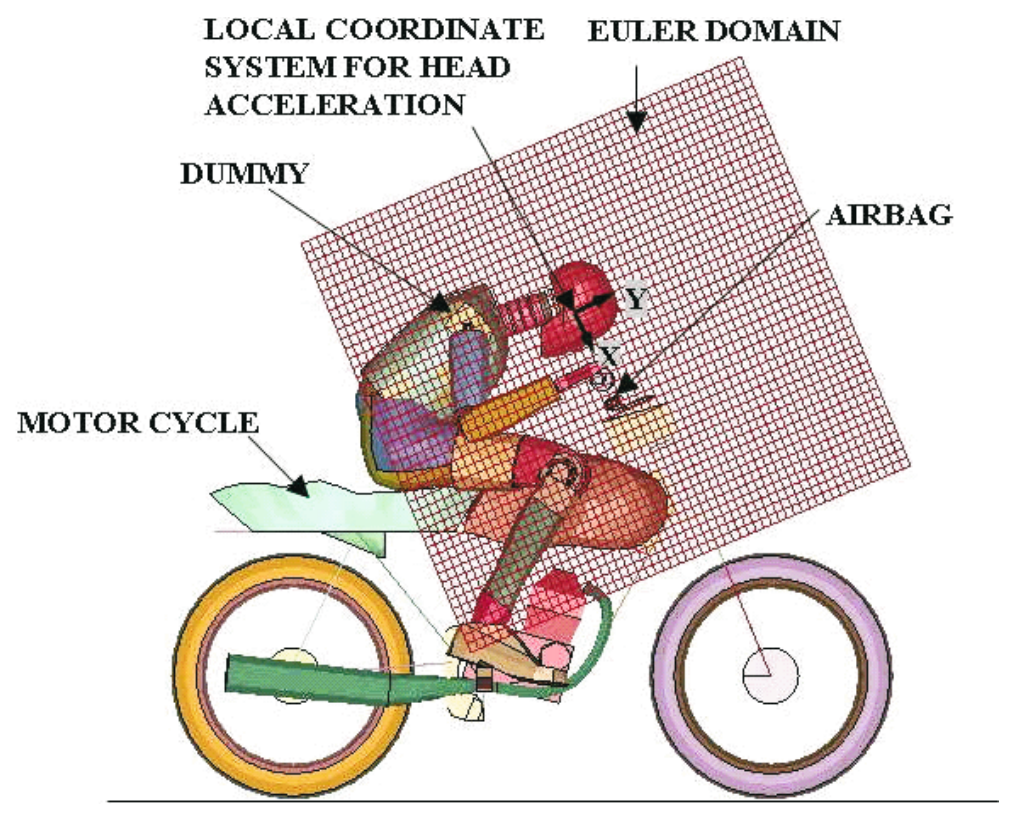

Figure 11. Set-up for the simulations for evaluating air bag performance. 


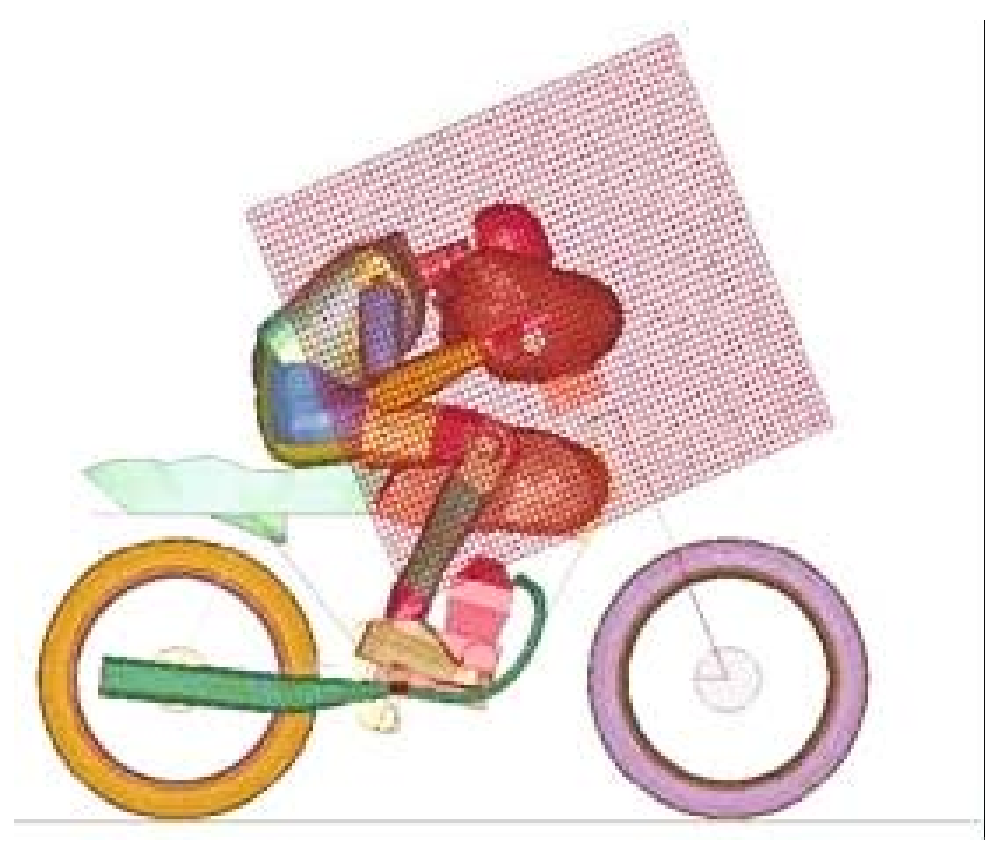

Figure 12. A typical inflated state of the motor cycle and the airbag.

Simulations were conducted for airbags of volume 1201 and 1801. with the distance of the nose of the dummy varying from $50 \mathrm{~mm}$ to $300 \mathrm{~mm}$. In all the cases, the dummy was initially stationary and the first contact with the airbag surface was during the deployment phase. A typical inflated state is shown in figure 12. Figures 13 and 14 show the variation of head acceleration in $x$ and $y$ directions for an airbag of volume of 120 liters with the distance between airbag and dummy varying from $50 \mathrm{~mm}$ to $150 \mathrm{~mm}$. Figures 15 and 16 show similar variations of head acceleration in $x$ and $y$ directions for the airbag volume of 180 liters.

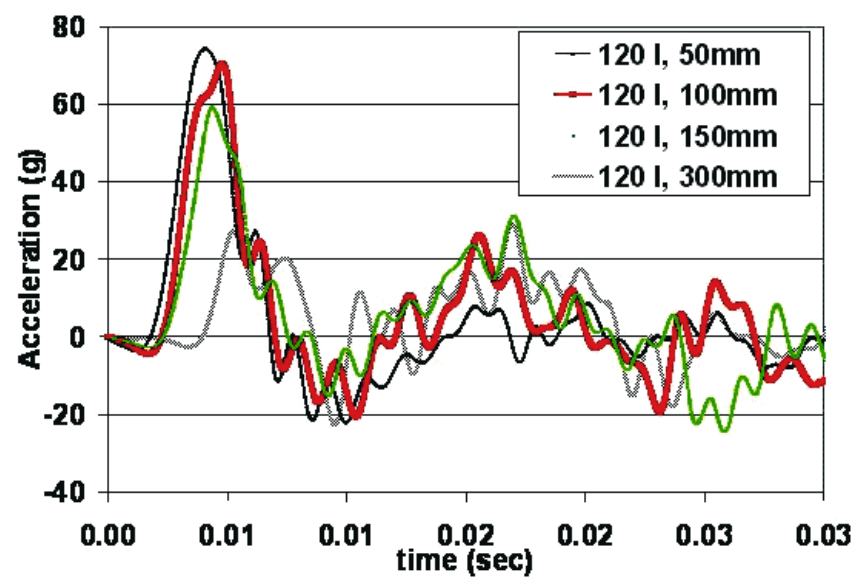

Figure 13. Variation of head $x$ acceleration with dummy position for 1201 airbag. 

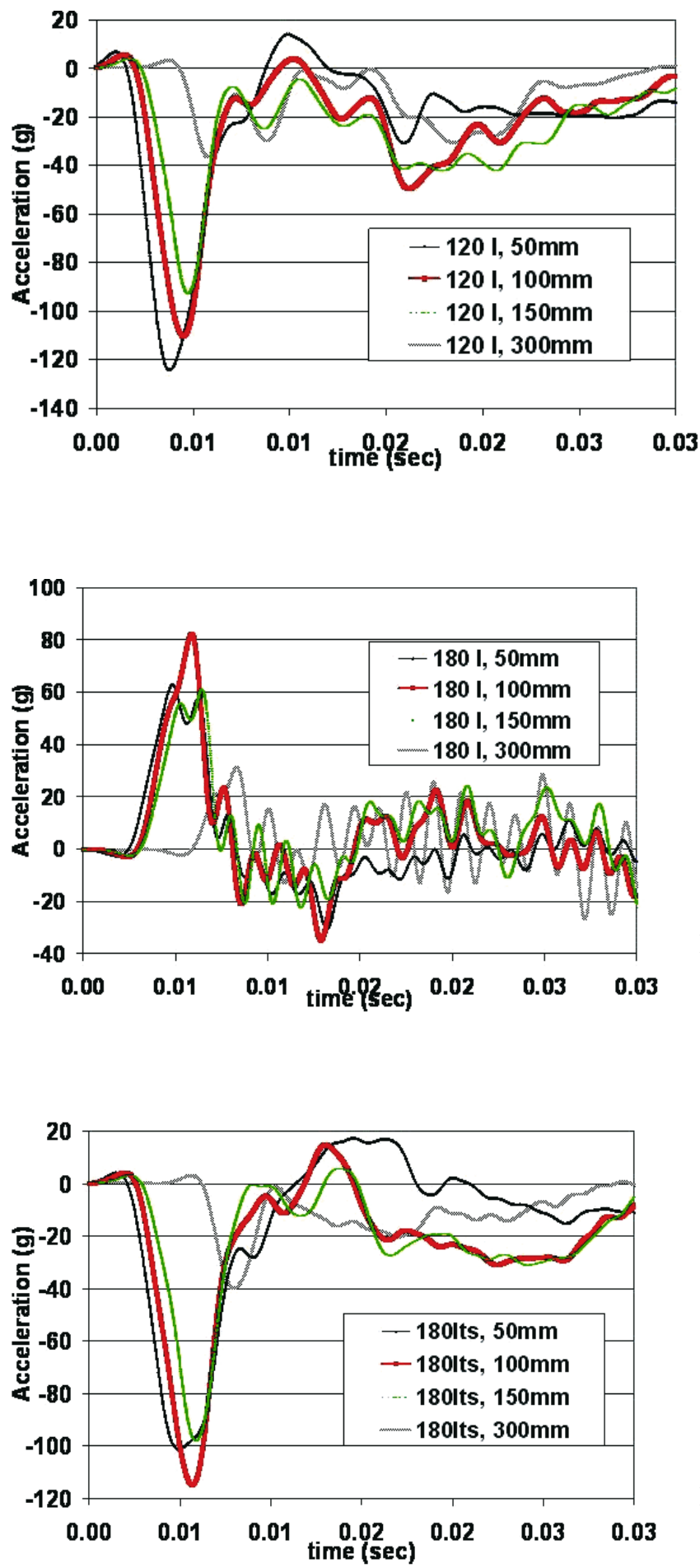

Figure 14. Variation of head $y$ acceleration with dummy position for 1201 airbag.

Figure 15. Variation of head $x$ acceleration with dummy position for 1801 airbag.

Figure 16. Variation of head $y$ acceleration with dummy position for 1801 airbag. 
Table 1. Initial material properties of soft tissue and top platen.

\begin{tabular}{ccccc}
\hline \multicolumn{5}{c}{ Soft tissue represented as linear visco-elastic material } \\
\hline $\begin{array}{ccccc}\text { Bulk modulus } \\
(\mathrm{K})\end{array}$ & $\begin{array}{c}\text { Short-term shear } \\
\text { modulus }\left(G_{0}\right)\end{array}$ & Long-term shear & Decay constant & Density \\
$\mathrm{Pa}$ & $\mathrm{Pa}$ & $\mathrm{Pa}$ & $(\beta)$ & $(\rho)$ \\
\hline 250000 & 115000 & 86000 & $s^{-1}$ & $\mathrm{~kg} / \mathrm{m}^{3}$ \\
\hline
\end{tabular}

Figures 13-16 show that as the initial distance between airbag and dummy head was increased, the peak head acceleration decreased. The variation in peak acceleration is only $20 \%$ when the distance changes from 50 to $150 \mathrm{~mm}$. When the distance changes to $300 \mathrm{~mm}$, the peak acceleration falls to $25 \%$ of the value at $50 \mathrm{~mm}$. A comparison of figure 13 with figure 15 and of figure 14 with figure 16 shows less than $10 \%$ increase in peak accelerations with $50 \%$ increase in airbag volume as a $100 \mathrm{~g} 4 \mathrm{~ms}$ pulse as observed here has the energy equivalent of a drop from a height of $0.5 \mathrm{~m}$. The $\mathrm{MC}$ rider helmets are designed to absorb larger impacts. The simulations done using a helmeted rider also confirmed this. The class of injury sustained by the rider due to contact with the airbag during its deployment phase has a low injury probability and variation due to the initial distance and the airbag size variation for practical airbags is not significant.

The effect of introducing a helmet and backing surface for the airbag, as well as effect of MC velocity on the head/neck injury are currently being studied. The study is limited by the fact that only one type of airbag (same folding pattern, single chamber) was used in the study. Variations caused by change in folding schemes for the airbag as well as larger variations in the position of the rider may be studied in the future.

\section{Conclusions}

The complexity in predicting injury probability of motor cycle riders is more in comparison to car occupants because of the greater number of possible interactions. These complexities are often not effectively represented by lumped mass or multi-body models and need FE modelling to reproduce some of these complexities. The methodology used by us Chawla et al (2001) for developing and then validating the FE model of the MC leading to high fidelity simulation has been presented.

The ISO 13232 specifies procedures to be followed for developing these computer simulations as well as for evaluating the effectiveness of safety devices fitted on the MC based on a cost-benefit analysis. Mukherjee et al (2001); Chawla et al (2003, 2004, 2004a, 2004b) have conduced simulation studies on the basis of the seven ISO13232 impact configurations. The kinematics of the motorcycle, rider and helmet are reproduced in the simulations Chawla et al (2004). The simulations point to issues that need to be resolved in car-MC impact simulations and the fidelity of the MATD dummy being used Mukherjee et al (2001), Chawla et al (2004).

ISO 13232 recommends a quantitative comparison of acceleration time histories and injury indices to establish the correctness of simulations. For dynamic simulations of this type, a match of the kinematics, even if qualitative, with regard to the key point and sequence of impacts and dummy configurations is a necessary step in validating the simulations. A correlation (in the absence of such a matching kinematics and points of impact) over the entire 
acceleration time history and the injury indices as proposed by ISO13232 may not be an effective indicator of the fidelity of simulations.

A study into the suitability of the airbags in small $100 \mathrm{cc}$ MCs commonly used in India using models validated using ISO13232 standards has been initiated. The ALE approach was seen to correctly predict experimentally observed airbag deployment sequences. The class of injury sustained by the rider due to contact with the airbag during its deployment phase is predicted to have a low injury probability and variation due to the initial distance and the airbag size variation for practical airbags is not significant. Hence airbags can be designed for motorcycle safety without great concern for inflicting injury to out-of-position riders.

The authors are thankful for the continuous support from the Japan Automobile Research Institute, and the Volvo Research and Educational Foundations.

\section{References}

Anonymous 1996 Motorcycles - test and analysis procedures for research evaluation of rider crash protective devices fitted to motorcycle ISO 13232, Geneva

Bothwell P W, Petersen H C 1971 Dynamics of motorcycle impact Vol. I, DOT HS 800586

Bothwell P W, Knight R E, Petersen H C 1973 Dynamics of motorcycle impact 1971-1973, Vol. I, DOT HS 800906

Canaple B et al 2002 Impact model development for the reconstruction of current motorcycle accidents. I. J. Crash 7(3): 307-320

Chawla A et al 2003 FE simulations of motorcycle-car frontal crashes, validation and observations. Proc. 18th ESV Conference (Nagoya, Japan)

Chawla A et al 2001 A methodology for car-motorcycle crash simulation. JARI Res. J. 23(2): 18-21

Chawla A, Mukherjee S, Mohan D, Bose D, Rawat P, Nakatani T, Sakurai M 2004 FE simulations of motorcycle car frontal crashes, validations and observations. Int. J. Crashworthiness 10(4): 319326

Chawla A, Mukherjee S, Sharma A 2004a Mesh generation for folded airbags. Computer Aided Design and Applications. 1(1): 269-276

Chawla A, Mukherjee S, Sharma A 2004b Mesh generation of folded airbags. Proceedings of the International CAD conference (Pattaya, Thailand)

Chawla A, Mukherjee S, Mohan D, Bose D, Rawat P, Sakurai M, Nakatani T 2004c FE simulations of car - motor cycle frontal crashes. JARI Res. J. 26(10): 37-43

Chawla A, Bhosale P, Mukherjee S 2005 Meshing of folded passenger side airbag mesh. Proceedings of SIAT'05 (Pune)

Chinn B P, Hope P D 1987 Protecting motorcyclists' legs. Proc. 11 th International technical conference on the enhanced safety of vehicles (Washington DC)

Chinn B P, Happian-Smith J, Macaulay M A 1989 The effect of leg protecting fairings on the overall motion of a motorcycle in a glancing impact. Int. J. Impact Engg. 8(3): 265-279

Chinn B P, et al 1996 Development and testing of a purpose built motorcycle airbag restraint system. Proc. 15th International technical conference on the enhanced safety of vehicles (Melbourne, Australia)

Cirak F, Radovitzky R 2003 A new Lagrangian-Eulerian shell fluid coupling algorithm based on level sets, Proceedings of the 44th AIAA/ASCE/ASME/AHS Structures, Structural Dynamics, and Materials Conference, Norfolk, VA, April

Deguchi M 2003 Modelling of a motorcycle for collision simulation. Proc. 18th International technical conference on the enhanced safety of vehicles (Tokyo, Japan) 
Happian-Smith J, Macaulay M A, Chinn B P 1987 Motorcycle impact simulation and practical verification. Proc. 11th International technical conference on the enhanced safety of vehicles (Washington DC)

Happian-Smith J, Chinn B P 1990 Simulation of airbag restraint systems in forward impacts of motorcycles. SAE 900752

Haufe A, Weimar K, Gohner U 2004 Advanced airbag simulations using fluid-structure-interaction and the Eulerian method in LS-DYNA, 1st NAFEMS CFD-Seminar, Wiesbaden, Germany, April

Iijima S, et al 1998 Exploratory study of an airbag concept for a large touring motorcycle Proc. 16th ESV Conf. (Ontario, Canada)

Kebschull S K, et al 1998 Injury risk/benefit analysis of motorcyclist protective devices using computer simulation and ISO 13232. Proc. 16th ESV Conf. (Ontario, Canada)

Knight R E, Petersen H C 1971 Dynamics of motorcycle impact, Vol III. DOT HS 800588

Knight R E, Petersen H C 1973 Dynamics of motorcycle impact, Vol III. DOT HS 800908

Knight R E, Peterson H C 1976 Dynamics of motorcycle impact, Vol III. Denver Research Inst

Kuroe T, Namiki H, Iijima S 2005 Exploratory study of an airbag concept for a large touring motorcycle: Further research second report, Proceedings of the ESV conference, paper No 05-0316

Marklund P-O, Nilsson L 2002 Simulation of airbag deployment using a coupled fluid structure approach, 7th International LS-DYNA Users Conference, Detroit, USA

Mukherjee S, Chawla A, Mohan D, Singh M, Sakurai M, Nakatani T 2000 Motor cycle wall crash, simulation and validation. Proc. of Pam Users Conference Asia, (Tokyo, Japan)

Mukherjee S et al 2001 Motorcycle-car side impact simulation. Proc. IRCOBI, (Isle of Man, UK)

Nakatani T et al 2001 A methodology for motorcycle-vehicle crash simulation - development of motorcycle computer simulation model. JSAE 20015417

Namiki H, Nakamura T, Iijima S 2003 A computer simulation for motorcycle rider-motion in collision. SAE 2003-32-0044

Nieboer J J et al 1993 Motorcycle crash test modelling. Proc. 37th STAPP Car Conf. (Warrendale, USA)

Rogers N M 1994 Evaluation of TRL designed leg protectors for a medium sized sport motorcycle. Proc. 16th ESV Conf. (Ontario, Canada)

Rogers N M, Zellner J W 2001 Factors and status of motorcycle airbag feasibility research, Seventeenth international technical conference on experimental safety of vehicles

Rogers N, Zellner J, Chawla A, Nakatani T 2005 Methodologies for motorcyclist injury prevention by use of computer simulations. Proc of ESV Paper No 05-0311-W (Washington DC)

Sporner A 1982 Experimentelle und mathematische simulation von motor-radkollisionen im vergleich zum realen unfallgschehen. $\mathrm{PhD}$ Dissertation (TU München)

Takatori O 1999 Deformation characteristics of tires on frontal collisions and applications to crash simulations. JSAE proceedings No. 10-99 17-20

Van Driessche H 1994 Development of an ISO standard for motorcycle research impact test procedures. Proc. 14th ESV Conf. (Munich, Germany)

Wang Y, Sakurai M 1999 Development and verification of a computer simulation model of motorcycleto-vehicle collisions. SAE 1999-01-0719

Watson P M, Donne G L 1989 ESM-4: A lightweight safety motorcycle, Twelth international technical conference on experimental safety of vehicles

Yettram A L et al 1994 Computer simulation of motorcycle crash tests. Proc. 4th ESV Conf. (Munich, Germany)

Zellner J W, Newman J A, Nicholas M 1994 Preliminary research into the feasibility of motorcycle airbag systems. Proc. 14th ESV Conf. (Munich, Germany) 